\title{
Geodesic Approach for an Efficient Trajectory Planning of Mobile Robot Manipulators
}

\author{
Pradip Kumar Sahu \\ Department of Industrial Design \\ National Institute of Technology Rourkela \\ Rourkela-769008, Odisha, India \\ Corresponding author: pradipsahu2@gmail.com \\ Bibhuti Bhusan Biswal \\ Department of Industrial Design \\ National Institute of Technology Rourkela \\ Rourkela-769008, Odisha, India
}

(Received April 27, 2019; Accepted July 24, 2019)

\begin{abstract}
In this paper, the geodesic approach has been employed for an effective, optimal, accurate and smooth trajectory planning of a mobile robot manipulator mechanism. Generally, geodesic can be described as the shortest curvature between two loci on a Riemannian manifold. In order to attain the planned end-effector motion, Riemannian metrics has been consigned to the forward kinematics of mobile robot wheel as well as the mobile robot manipulator workspace. The rotational angles of wheel and joint kinematic parameters are chosen as local coordinates of spaces to represent Cartesian trajectories for mobile wheel rotation trajectories and joint trajectories respectively. The geodesic equalities for a given set of boundary conditions are evaluated for the chosen Riemannian metrics and the computational results of the geodesic equations have been shown. So as to verify and validate the efficiency of the chosen geodesic scheme, the method has been implemented for the motion planning and optimization of a mobile robot with a simple $3 \mathrm{R}$ manipulator installed upon its platform.
\end{abstract}

Keywords- Mobile robot, Trajectory planning, Homogeneous transformation, Geodesic method, Motion analysis.

\section{Introduction}

Nowadays, there have been tremendous applications of mobile robots in various fields of manufacturing, assembly, inspection, space exploration, mining, marine, education, etc. The mobile robots in recent times are acting as a slave for human in helping and assisting them with numerous works of simple nature to very difficult types. In critical and uncontrollable areas like space exploration, mining, marine, etc., the motion planning of a mobile robot and its manipulator is challenging and tough. Therefore, the demand arises for optimal motion planning of a mobile robot and its manipulator end effector. The optimal motion planning mainly focuses in providing an efficient trajectory of the manipulator and wheel for the desired task persistently with more optimality, accuracy and smoothness. The NP hard problem of manipulator inverse kinematics and this has to be a difficult task due to the evaluation of very lengthy equations. Most often the solution to IK problem will result in multiple solutions. Sometimes, the IK solution may not be a closed form solution due to the singularities and nonlinearities convergence nature and it is very hard to compute. Generally, the actuators simulations are performed in the joint space and the end-effector tasks are always accomplished in the Cartesian space. Therefore, the demand arises for the study of the robot work volume, trajectory generation and optimization, motion control, etc. 
International Journal of Mathematical, Engineering and Management Sciences

Vol. 4, No. 5, 1196-1207, 2019

https://dx.doi.org/10.33889/IJMEMS.2019.4.5-094

\section{Related Work}

The problem of robotic manipulator trajectory planning, mobile robot motion planning and its optimization have been investigated by numerous academics and researchers. In order to tackle the trajectory planning problem, polynomial interpolation along with genetic algorithm has been used (Eldershaw and Cameron, 2000). Similarly, the genetic algorithm approach was employed to perform the same task (Yun and Xi., 1996; Tian and Collins., 2004). An optimal motion configuration was suggested for robotic manipulators trajectory planning (Zha, 2002). Later on, the task of optimal trajectory tracking and control has been carried out for an automated material handling process (Zha and Chen, 2004). A novel scheme of trajectory planning was suggested which considered continuous machining (Olabi et al., 2010). A parametric speed interpolator came into the picture, which results in a smooth trajectory. Multi degree Splines was introduced to trajectory planning methods (Liu et al., 2013). When the motion actually performed in the joint space by the end-effector through interpolating sequence of via points, it is not easily predictable. The nonlinearity of the inverse kinematics may indicate an uneven joint motion in joint space. In order to achieve a smooth motion in Cartesian space, the data obtained from the interpolation scheme are plotted in joint space. Subsequently, an uneven and imprecise motion could be acquired in Cartesian space.

The dynamic characteristics of the 2DOFs robot in 3D space have been analyzed (Rodnay and Rimon, 2001). They signified trajectories by allowing the manipulator to perform the free movement by introducing geodesics on the dynamic surface. Lie group approach has been chosen to generate trajectories resulting from non-linear motion (Zefran et al., 1998). They chose Lie group and consequently defined a left invariant Riemannian metric on it to generate smooth trajectories. In order to control the manipulators motion along helical trajectories a Lie group method has been implemented (Selig and Ovseevitch, 1996). The employed method for the planning was found to be complicated, abstract, and that can be not put into real practice easily. The geodesic approach has been employed for optimal and efficient trajectory planning of industrial manipulators (Zhang et al., 2007). They have allocated Riemannian metric for the kinetic energy and arc length to obtain optimal and precise path. However, this method had some shortcomings which decreased the credibility of that method by considering only less than 3 DOFs manipulators for trajectories optimization and the orientation problem has not been addressed. The efficacy of geodesic based trajectory planning was described for a PUMA 560 robotic manipulator and the simulation results verify their purpose of implementation (Chen et al., 2014). The geodesic motion is accomplished for a set of given end condition by assigning Riemannian metrics to the workspace.

An efficient and optimal trajectory has been performed for a 4-DOF SCARA manipulator by employing geodesic approach (Jena et al., 2016). The depicted simulation results obtained from the geodesic computation confirmed the efficacy of the implemented method. The geodesic technique was used for optimal, accurate and smooth trajectory planning of a 6R Kawasaki robot industrial robot (Sahu, 2016). Here, a combined Riemannian metric has been assigned for the orientation and position of the robot manipulator workspace. A comparative analysis was carried out among four meta-heuristic algorithms namely bat algorithm, firefly algorithm, PSO algorithm and TLBO algorithm for the end-effector motion planning of a SCARA manipulator (Sahu et al., 2019). The comparison of simulations confirms the superiority of TLBO algorithms over the other considered methods for the trajectory planning purpose. 
International Journal of Mathematical, Engineering and Management Sciences

Vol. 4, No. 5, 1196-1207, 2019

https://dx.doi.org/10.33889/IJMEMS.2019.4.5-094

Earlier the geodesic method has been used for the purpose of end-effector trajectory planning of fixed or stationary industrial robotic manipulator. However, the current work is focused upon using geodesic scheme for an optimal and efficient motion planning for a mobile robot with a manipulator. The present work also includes the motion planning of wheel of the mobile robot along with end-effector trajectory optimization installed on the mobile robot platform. In order to obtained the geodesic condition or formulae for the desired linear motion, the forward kinematic of the robot wheel and the manipulator end-effector has been assigned with Riemannian metrics. Firstly, wheel rotation parameters and joint parameters are considered as local coordinates of position space. Then, geodesic has been obtained from mathematical formulation followed by obtaining the wheel rotations trajectories, joint trajectories and characterizing Cartesian trajectories by joint trajectories.

\section{Mathematical Modeling using Geodesic}

Geodesic can be described as the smallest curvature between two points on any Riemannian surface or manifold. Any abstracted arbitrary surface or manifold is called as Riemannian surface. The characteristic of geodesic is such that the velocity along its curvature remains the constant (5). The shortest route through a Riemannian surface joining any two loci is defined as geodesic. It has another property that velocity along this geodesic curve remains in-variant (5). The comprehensive background of geodesic has been presented in the following sections.

\subsection{Riemannian Manifold}

A simple manifold ' $\mathrm{M}$ ' ' can be defined as a Hausdroff topological space and in that space any point ' $p$ ' has an area $A \subset \mathrm{M}^{\mathrm{n}}$, topologically isomorphism with ' $\mathrm{R}^{\mathrm{n}}$ ', the Euclidean space. Hence, it can be given by a function as depicted below;

$\Phi: \mathrm{A} \rightarrow \Phi(\mathrm{A}) \subset \mathrm{R}^{\mathrm{n}}$

Similarly, a Riemannian manifold can be represented by $\left(\mathrm{M}^{\mathrm{n}}, \mathrm{g}\right)$. Here, ' $\mathrm{M}^{\mathrm{n}}$ ' signifies an $\mathrm{n}$ dimensional differentiable topological space and ' $\mathrm{g}$ ' defines the Riemannian metric. The main properties of Riemannian metric are symmetric, positive and definite quadratic form. Generally, the distance along the chosen manifold is considered as the Riemannian metric. Let us assume any neighborhood area ' $\mathrm{A}$ ' of a point in the manifold and the local co-ordinates be $\theta_{1}, \theta_{2}, \theta_{3}, \ldots$.. $\theta_{n}$, then the Riemannian metric can be given by (2);

$g=\sum_{i, j=1}^{n} g_{i j} d \theta_{i} d \theta_{j}$

where, $g_{i j}=g\left(\frac{\partial}{\partial \theta_{i}}, \frac{\partial}{\partial \theta_{j}}\right)$.

Let us assume a curve (3) on a Riemannian surface,

$\theta_{i}=\theta_{i}(t)$

Therefore, the tangent vector of the curve defined in equation (3) is given by,

$T_{i}=\sum_{i=1}^{n} d \theta_{i} \frac{\partial}{\partial \theta_{i}}$

If the arc length is considered as the Riemannian metric in the chosen manifold, then the geodesic condition (5) may be reached by equating the tensor (4) along the tangent vector of the manifold 
International Journal of Mathematical, Engineering and Management Sciences

Vol. 4, No. 5, 1196-1207, 2019

https://dx.doi.org/10.33889/IJMEMS.2019.4.5-094

with zero. Hence, the geodesic formulation of the space under consideration can be defined as shown in equation (5).

$\frac{d^{2} \theta_{i}}{d t^{2}}+\tau_{k j}^{i} \frac{d \theta_{k}}{d t} \frac{d \theta_{j}}{d t}=0$

Here, $\tau_{k j}^{i}$ signifies Christoffel symbol and $\tau_{k j}^{i}$ is evaluated by equation (6) as given below,

$\tau_{k j}^{i}=\frac{1}{2} g^{m i}\left(\frac{\partial g_{k m}}{\partial \theta_{j}}+\frac{\partial g_{j m}}{\partial \theta_{k}}-\frac{\partial g_{k j}}{\partial \theta_{m}}\right)$

where, $g^{m i}$ symbolizes one of the entries of the Riemannian coefficient matrix inverse.

\subsection{Geodesic Methodology to Trajectory Planning}

The DH demonstration of manipulator systems helps in identifying the DH kinematic parameters. As the links are the product of successive joints, the basic homogeneous transformation matrix, ${ }_{i}^{i-1} T$ for any link may be calculated by equation (7) as written below;

$$
{ }_{i}^{i-1} T=\left[\begin{array}{cccc}
c \theta_{i} & -s \theta_{i} & 0 & a_{i-1} \\
s \theta_{i} c \propto_{i-1} & c \theta_{i} c \propto_{i-1} & -s \propto_{i-1} & -d_{i} s \propto_{i-1} \\
s \theta_{i} s \propto_{i-1} & c \theta_{i} s \propto_{i-1} & c \propto_{i-1} & d_{i} c \propto_{i-1} \\
0 & 0 & 0 & 1
\end{array}\right]
$$

here, $\quad a_{i}=$ length of link and $d_{i}=$ offset distance of $i^{\text {th }}$ joint

$s \theta_{i}=\sin \theta_{i}, c \theta_{i=} \cos \theta_{i}, \theta_{i}=$ the rotation angle,

$s \alpha_{i=} \sin \alpha_{i}, c \alpha_{i=} \cos \alpha_{i}, \alpha_{i}=$ twist angle.

Let us say, ' $\mathrm{G}$ ' is the global frame and ' $\mathrm{C}$ ' is the frame fixed on the centroid of the mobile robot platform. Thus, the transformation matrix for the movement from the frame, $\mathrm{G}$ to the frame, $\mathrm{C}$ can be determined as given in equation (8).

${ }_{C}^{G} T=\left(\begin{array}{cccc}c \Phi & -s \Phi & 0 & X_{c} \\ s \Phi & c \Phi & 0 & Y_{c} \\ 0 & 0 & 1 & Z_{c} \\ 0 & 0 & 0 & 1\end{array}\right)$

here, $\Phi$ signifies the heading angle of the mobile platform, $c \Phi=\cos \Phi, s \Phi=\sin \Phi$ and $\left(\mathrm{X}_{c}, \mathrm{Y}_{\mathrm{c}}\right.$, $\mathrm{Z}_{\mathrm{c}}$ ) are the coordinates of the centroid of the mobile platform.

Let ' $\mathrm{O}$ ' be the first frame, upon which the robotic manipulator system is fixed. So, the relative position among frame-O and frame-C is unaffected, as the manipulator stands fixed over the mobile platform. And hence, the transformation matrix between that two frame ' $\mathrm{O}$ ' and frame ' $\mathrm{C}$ ', can be calculated as given in equation (9);

$$
{ }_{O}^{C} T=\left(\begin{array}{cccc}
1 & 0 & 0 & -d \\
0 & 1 & 0 & 0 \\
0 & 0 & 1 & h \\
0 & 0 & 0 & 1
\end{array}\right)
$$


International Journal of Mathematical, Engineering and Management Sciences

Vol. 4, No. 5, 1196-1207, 2019

https://dx.doi.org/10.33889/IJMEMS.2019.4.5-094

here, ' $d$ ' and ' $h$ ' denotes the distances between ' $C$ ' and ' $O$ ' in X-direction and Z-direction respectively.

Accordingly, the final forward kinematics of the manipulator end-effector may be computed by equation (10),

end-effector $T={ }_{C}^{G} T \times{ }_{0}^{C} T \times{ }_{1}^{0} T \times{ }_{2}^{1} T \times \ldots \ldots . .{ }_{n}^{n-1} T$

Let the final end-effector homogeneous transformation matrix, $\mathrm{T}$ of a robot end-effector as follows,

$$
\underset{\text { end-effector }}{\text { global }} T=\left(\begin{array}{cc}
R & P \\
0 & 1
\end{array}\right)\left(\begin{array}{cccc}
n_{x} & o_{x} & a_{x} & p_{x} \\
n_{y} & o_{y} & a_{y} & p_{y} \\
n_{z} & o_{z} & a_{z} & p_{z} \\
0 & 0 & 0 & 1
\end{array}\right)
$$

here, $P$ signifies the end-effector position vector $\left(p_{x}, p_{y}, p_{z}\right)$ and $R$ denotes the end-effector orientation vector $\left(n_{s}, o_{s}, a_{s}\right)$.

\subsection{Use of Geodesic for Trajectory Planning}

Let the distance be assigned with Riemannian metric for a Euclidean space. Hence, the Riemannian metric chosen as the distance metric can be described as depicted in equation (12),

$g_{p}=(d P)^{2}$

here, $d \mathrm{P}$ is termed as the first derivative of $P=\left(p_{x}, p_{y}, p_{z}\right)$.

\subsection{Riemannian Metric Selection Procedure}

The main job in motion planning using geodesic is to assign appropriate Riemannian metric for the planning and optimization of the desired motion under consideration. The selected metric should possess the symmetric and positive definite quadratic form. At the same time, the metric has to be shown in terms of distance and other parameters which are related to the preferred/chosen motion. In this context, the Riemannian metric is formulated by the equation (13) as follows,

$g=\left(\begin{array}{lllll}d \theta_{1} & d \theta_{2} & d \theta_{3} & \ldots & \ldots d \theta_{n}\end{array}\right) G\left(\begin{array}{c}d \theta_{1} \\ d \theta_{2} \\ d \theta_{3} \\ \cdot \\ . \\ d \theta_{n}\end{array}\right)$

where, $G=\left(g_{i j}\right)_{n x n}$ and it is a coefficient matrix in reference to ' $g$ ' and $\theta_{i}(i=1 \ldots n)$ defines linear or angular joint displacements.

Firstly, the coefficient matrix ' $G$ ' is evaluated and after that the Christoffel symbols ' $\tau_{k j}^{i}$ ' are found out by equation (6). The calculated Christoffel symbols are put in equation (5) to formulate the geodesic equalities. For each joint variable there will be one geodesic equation. As the geodesic equations are second order simultaneous differential equations, they can be solved for 
International Journal of Mathematical, Engineering and Management Sciences

Vol. 4, No. 5, 1196-1207, 2019

https://dx.doi.org/10.33889/IJMEMS.2019.4.5-094

the chosen trajectory for a given set of end conditions. The solutions of the geodesic equations will results in the wheel rotation trajectories, the end-effector trajectories and joint trajectories for the considered movement.

\subsection{Mathematical Formulation and Numerical Computation of Geodesic for the Mobile Robot Manipulator}

Figure 1 depicts a wheeled mobile robot with a simple 3R spatial manipulator fixed on the mobile robot platform, which is selected for validation of the complete course of motion planning by implementing the geodesic method.

Assumptions: The assumptions made in this research are as follows:

a) The centroid of the mobile robot platform is assumed at the origin position, i.e. $(0,0,0)$.

b) The manipulator is assumed to be fixed in the mobile platform, at a distance (say $\mathrm{d}=1 \mathrm{~mm}$ ) from the centroid of the platform in X-direction and at a height (say $\mathrm{h}=1 \mathrm{~mm}$ ) above the platform in Z-direction.

c) The heading angle, ' $\Phi$ ' is also assumed as zero to simplify the computation.

d) The assumptions have been made that the masses and length of links are chosen as one.

e) The mass of all links is presumed to be concentrations at the endpoint of links/arms.

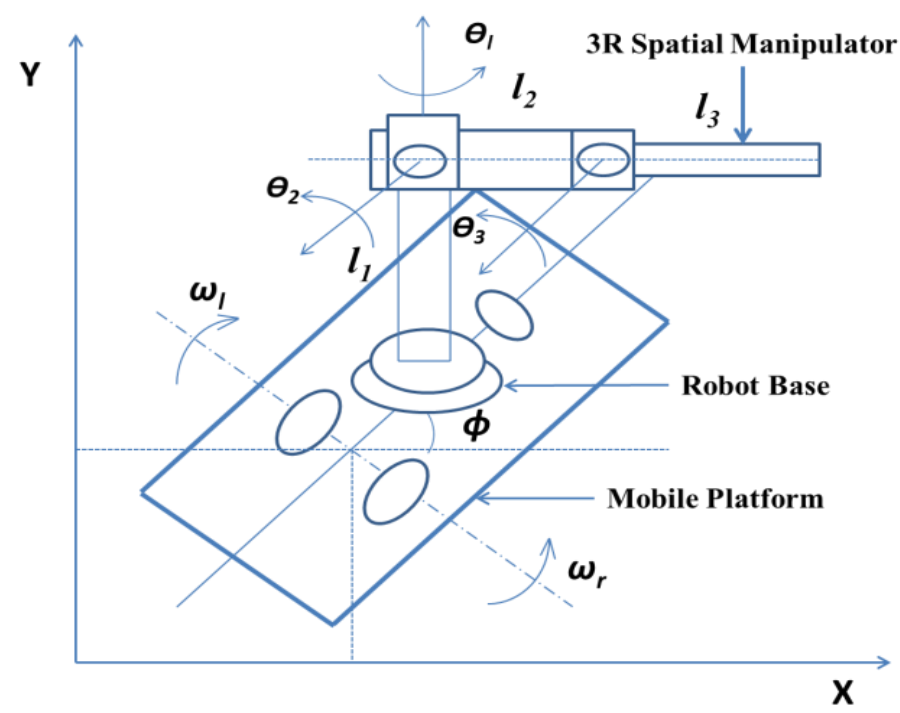

Figure 1. The kinematic model of a 3-DOF manipulator installed on the wheeled mobile robot platform

$\theta_{r}$ and $\theta_{l}$ are rotational angles of the right and left wheel respectively.

$\omega_{r}$ and $\omega_{l}$ are rotational velocities of the right and left wheel respectively.

$2 b=$ distance between the wheel and $\mathrm{r}=$ radius of wheel $(r=b=1$, assumed $)$

The kinematic equation of motion for the mobile robot wheel is evaluated as follows;

$X=-(r / 2) * \sin (\Phi) *\left(\theta_{r}+\theta_{l}\right)$

$Y=(r / 2) * \cos (\Phi) *\left(\theta_{r}+\theta_{l}\right)$

$\Phi=(r / b) *\left(\theta_{r}-\theta_{l}\right)$ 
International Journal of Mathematical, Engineering and Management Sciences

Vol. 4, No. 5, 1196-1207, 2019

https://dx.doi.org/10.33889/IJMEMS.2019.4.5-094

The position vector $P=(X, Y)$ is function of on $\theta_{l}$ and $\theta_{r}$ and the conforming Riemannian metric is given as follows;

$g_{p w}=(d P)^{2}=\left(\begin{array}{ll}d \theta_{r} & d \theta_{l}\end{array}\right) G\left(\begin{array}{l}d \theta_{r} \\ d \theta_{l}\end{array}\right)$

where, $d p$ is represented as the derivative of the position vector $P, G=\left(g_{i j}\right)_{2 \times 2}$ is the coefficient matrix associated with $g_{p w}$ and

$G=\left(g_{i j}\right)_{2 \times 2}=\left(\begin{array}{cc}\left(r^{2} / 4\right)\left(1+\left(\theta_{r}+\theta_{l}\right)^{2} * \mathrm{r}^{2} / \mathrm{b}^{2}\right) & 0 \\ 0 & \left.r^{2} / 4\right)\left(1+\left(\theta_{r}+\theta_{l}\right)^{2} * \mathrm{r}^{2} / \mathrm{b}^{2}\right)\end{array}\right)$

The geodesics can be evaluated from the two equations i.e. equation (5) and equations (6), for the Riemannian metric (16) and the considered end conditions. The geodesics conforming to $g_{p w}$ are second-order differential equations with two unknown parameters, $\theta_{l}$ and $\theta_{r}$.

The final transformation matrix equation for the robotic manipulator end-effector as obtained from equation (10) and (11) can be obtained as follows,

$$
\begin{aligned}
& p_{x}=c_{1} *\left(l_{2} * c_{2}+l_{3} * c_{23}\right)-1 \\
& p_{y}=s_{1} *\left(l_{2} * c_{2}+l_{3} * c_{23}\right) \\
& p_{z}=a_{2} * s_{2}+a_{3} * s_{23}+1
\end{aligned}
$$

here, $P_{x}, P_{y}$ and $P_{z}$ define the unit position vector $(P)$ of the end-effector of the robot. $l_{1}, l_{2}$ and $l_{3}$ denote the length of link 1,2 and 3 respectively. $\theta_{1}, \theta_{2}$, and $\theta_{3}$ represent the angle of joint 1,2 and 3 respectively. $c_{1}, c_{23}, s_{1}, s_{23}$ symbolizes $\cos \theta_{1}, \cos \left(\theta_{2}+\theta_{3}\right), \sin \theta_{1}, \sin \left(\theta_{2}+\theta_{3}\right)$ respectively.

The Riemannian metric tensor of the position vector $P$ can be defined as given below in the equation (18),

$$
g_{p m}=(d P)^{2}=\left(\begin{array}{lll}
d \theta_{1} & d \theta_{2} & d \theta_{3}
\end{array}\right) G\left(\begin{array}{l}
d \theta_{1} \\
d \theta_{2} \\
d \theta_{3}
\end{array}\right)
$$

here, $d \mathrm{P}$ represents the position vector derivative of $P, G=\left(g_{i j}\right)_{3 \times 3}$ defines the coefficient matrix for $g_{p m}$.

$G=\left(g_{i j}\right)_{3 \times 3}=\left(\begin{array}{ccc}\left(c_{2}+c_{23}\right)^{2} & 0 & 0 \\ 0 & 2+2 c_{3} & 1+c_{3} \\ 0 & 1+c_{3} & 1\end{array}\right)$

The geodesic equations are computed from the equation (5) and (6), for the aforesaid matrix equation (19). The second-order differential equations of geodesic possessing the unknown parameters, $\theta_{1}, \theta_{2}$ and $\theta_{3}$ have been acquired for the Riemannian metric ' $g_{p m}$ '. The geodesic is solved numerically by Runge-Kutta fourth step method by considering the assumed end conditions of the motion. 
International Journal of Mathematical, Engineering and Management Sciences

Vol. 4, No. 5, 1196-1207, 2019

https://dx.doi.org/10.33889/IJMEMS.2019.4.5-094

The current research assumes the motion to be a linear movement of end-effector between two selected points in the robot work volume. Accordingly, the robot wheel as well as the manipulator end-effector accomplishes a linear motion with reference to the attained geodesic. The sequential steps of motion planning using the geodesic scheme have been presented in Figure 2.

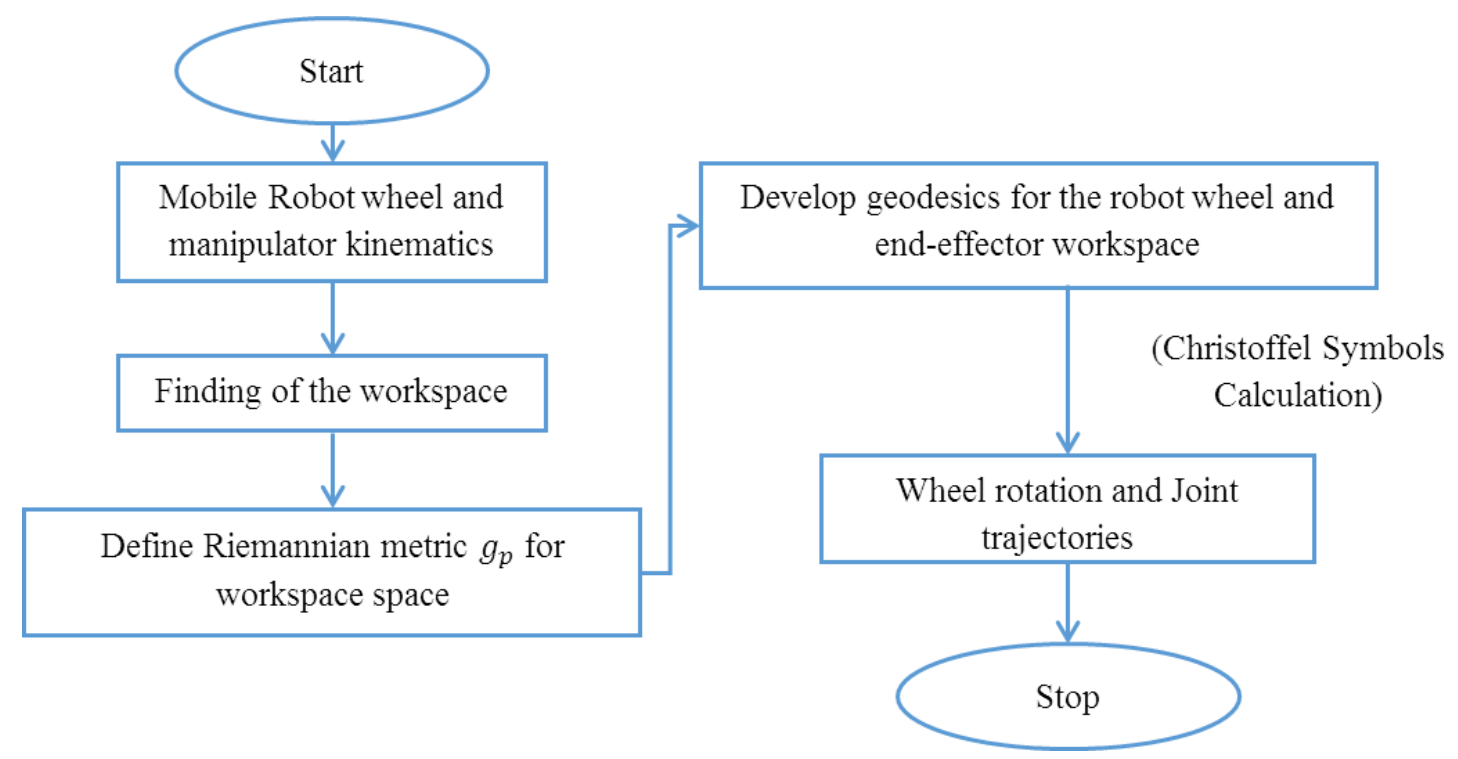

Figure 2. Flowchart showing the step by step process of mobile robot motion planning using the geodesic scheme

\section{Results of the Simulation and Discussion}

The wheel rotation trajectories and its byproducts like velocity, acceleration and jerk have been presented in Figure 3. The results confirm the optimality, smoothness and accuracy of the attained trajectories. The manipulator Cartesian position trajectory is chosen for the linear movement of end-effector from starting point, $\mathrm{P}_{1}(1.1422,0.0414,1.4506)$ to target point, $\mathrm{P}_{2}(0.9327,0.1968$, 1.5304). The end-effector Cartesian position trajectory for the selected motion between the two points is depicted in Figure 4. It can be evidently observed from Figure 4 that the obtained Cartesian trajectory (by using geodesic method for trajectory planning) is a straight line. Henceforth, the constant velocity of the end-effector along the geodesic line confirms the zero acceleration of end-effector motion. This outcome of straight line motion between any two points on any given workspace itself verifies the optimality of implemented techniques.

The corresponding manipulator joint trajectories for its position, velocity, acceleration, and jerk for the considered motion have been shown in Figure 5. Here, in Figure 5 it can also be confirmed that the manipulator joint trajectories of all the three joint angles are found to be optimal, accurate and smooth. Similarly, the accomplished derivatives of joint angles i.e. velocity, acceleration, and jerk are also found to be smooth and accurate. The overall simulation results of motion planning for the mobile wheel rotation as well as the end-effector trajectory optimization by employing the proposed geodesic approach favors the optimality, exactness, and smoothness the of the mobile robot motion. Therefore, an effective, efficient and optimal motion planning of a mobile robot with precision and smoothness can be performed by implementing a geodesic scheme. 
International Journal of Mathematical, Engineering and Management Sciences

Vol. 4, No. 5, 1196-1207, 2019

https://dx.doi.org/10.33889/IJMEMS.2019.4.5-094
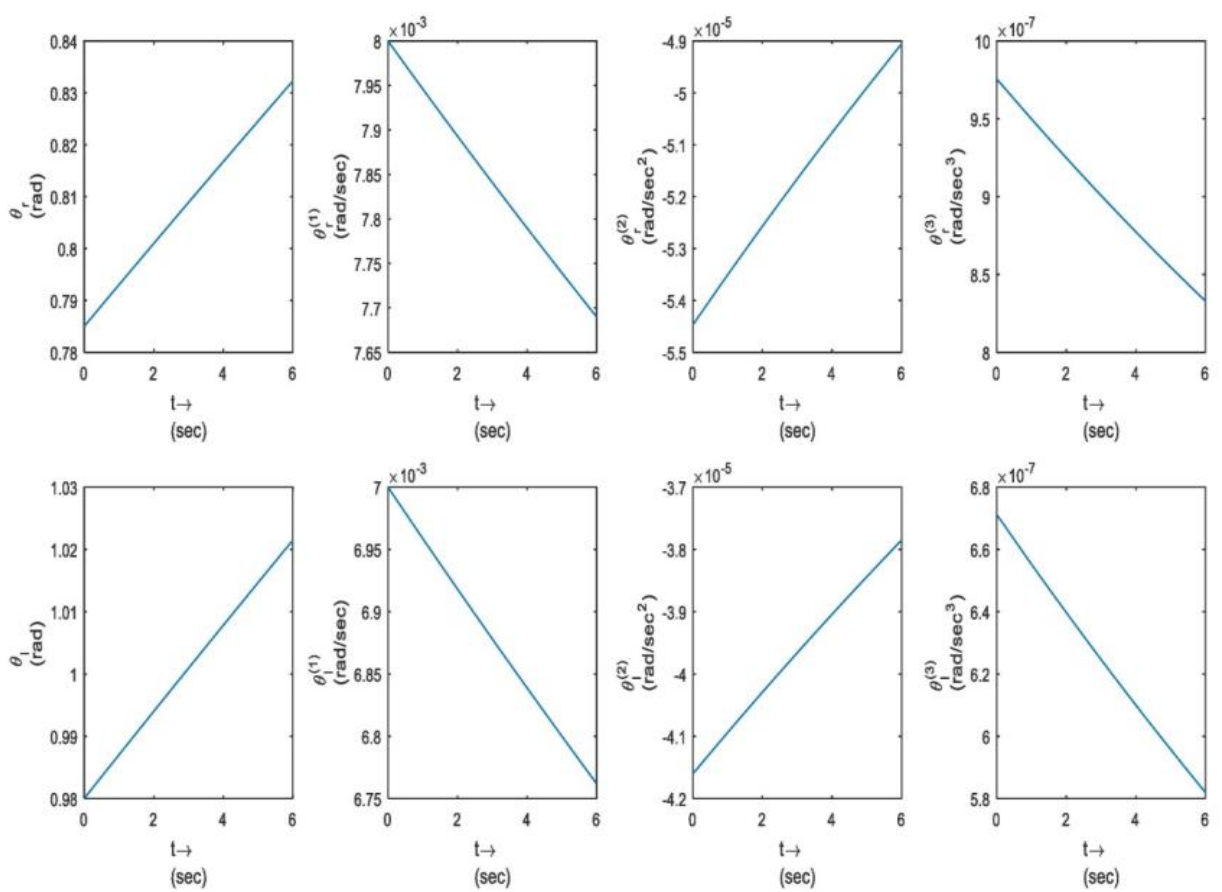

Figure 3. Trajectories of the wheel rotation angles and their derivatives

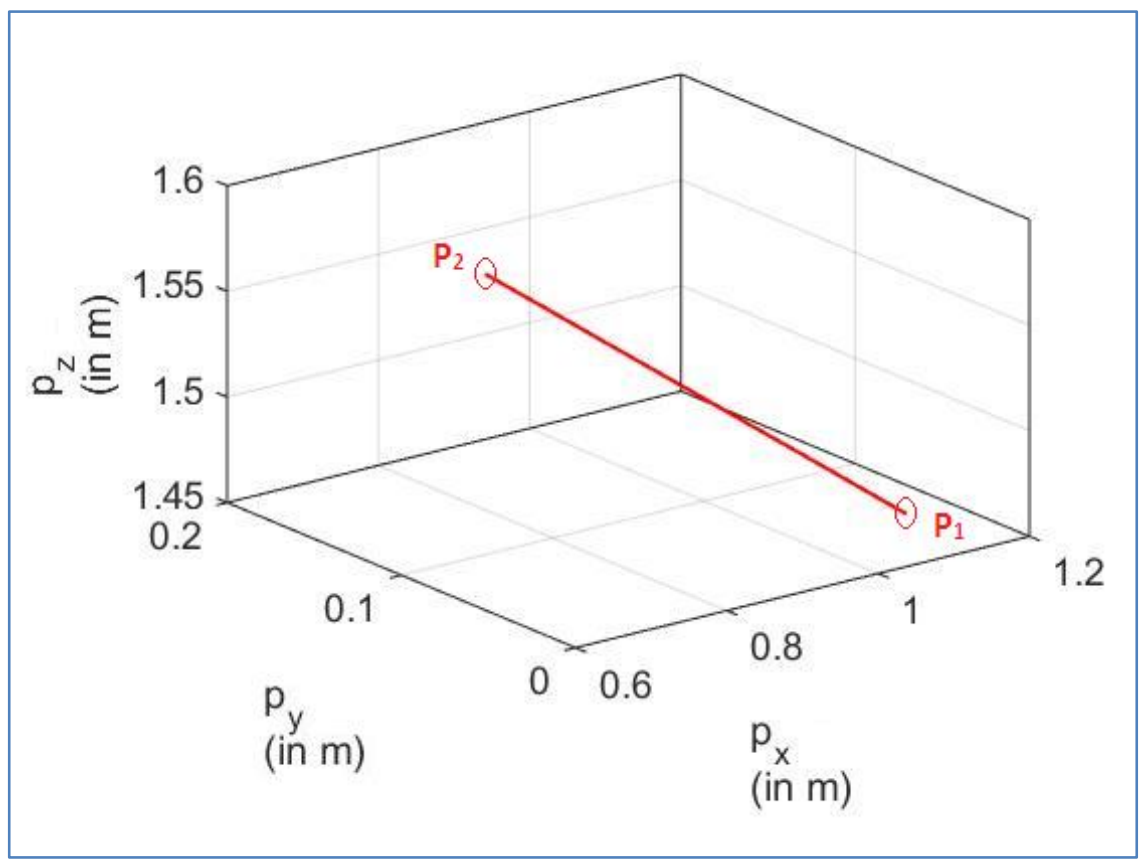

Figure 4. Mobile robot end-effector position trajectory 
International Journal of Mathematical, Engineering and Management Sciences

Vol. 4, No. 5, 1196-1207, 2019

https://dx.doi.org/10.33889/IJMEMS.2019.4.5-094

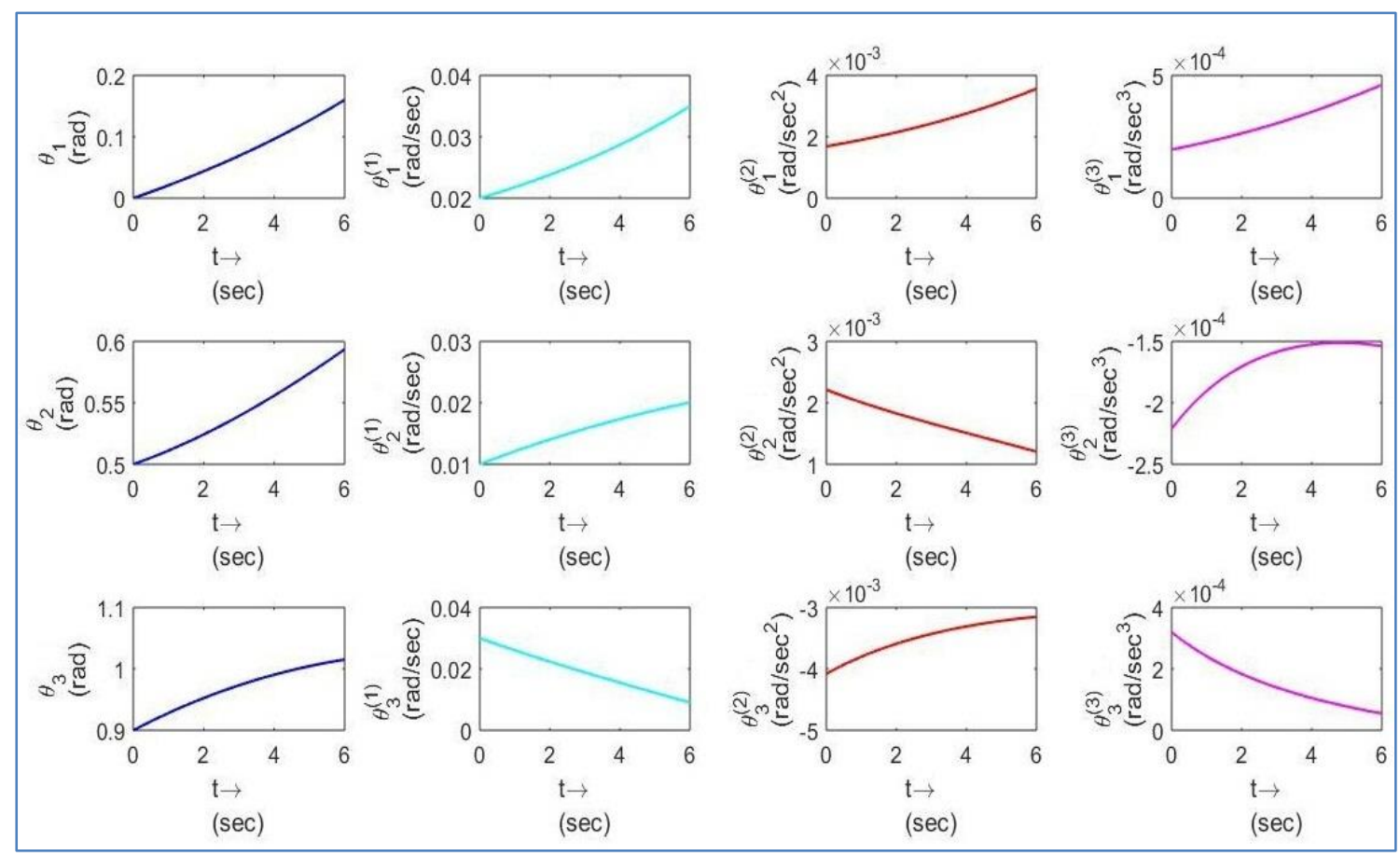

Figure 5. Joint trajectories of the end-effector and their derivatives

Geodesic is better than other mathematical and heuristic methods for optimal trajectory planning of robotic manipulator. Because other mathematical and heuristic methods provide multiple solutions and it need another tool for optimization purpose. However, unlike the other techniques, the geodesic method provides a unique and optimal solution. The geodesic is an inbuilt optimization tool and it does not require another tool for optimization. Therefore, the computational cost also becomes less.

\section{Conclusion}

An effective, optimal, precise and smooth motion planning of a mobile robot with manipulator link system has been accomplished by using the geodesic technique. The geodesic equalities have been evaluated for the considered linear motion of the mobile wheel and the manipulator, the forward wheel kinematics and manipulator forward kinematics (the workspace) has been assigned with Riemannian metrics. The geodesic equations are solved by using Runge-Kutta fourth step method. The numerical simulation results of different parameters of mobile robot as obtained from the geodesic evaluations have been presented. The suggested methodology and concept can have major impression in the field where a mobile robot is intended to move to a location away from its present position to perform certain task with the aid of the manipulator system. The existence of obstacles in between the source/starting and goal position, the geodesic should be replanned from starting position to obstacle position and simultaneously from the obstacle to the goal position. This makes the motion planning to accomplish a constant velocity profile with this in turn leads to the smoothness of the planned trajectory. The geodesic can be employed to a manipulator of more than three DOF with same higher optimality, accuracy and smoothness of the trajectory. However, with the increase in DOF of the manipulator, the computational cost will increase as the mathematical computation becomes more complex. 
International Journal of Mathematical, Engineering and Management Sciences

Vol. 4, No. 5, 1196-1207, 2019

https://dx.doi.org/10.33889/IJMEMS.2019.4.5-094

The present work assumed the heading angle as zero for mobile robot manipulator and the planned method has been implemented and analyzed for a simple 3R manipulator system. Also, the end-effector motion and the mobile robot motion are considered to be of linear type. Hence, the future work of this research aims at developing geodesic model and conditions for the trajectory optimization problem of mobile manipulator system of higher DOFs and also by introducing non-linear motion cases.

\section{Conflict of Interest}

The authors confirm that there is no conflict of interest to declare for this publication.

\section{Acknowledgment}

The authors would like to appreciate the effort from editors and reviewers. This research did not receive any specific grant from funding agencies in the public, commercial, or not-for-profit sectors.

\section{References}

Chen, Y., Li, L., \& Ji, X. (2014). Smooth and accurate trajectory planning for industrial robots. Advances in Mechanical Engineering, Volume 2014, Article ID 342137, 8 page.

Eldershaw, C., \& Cameron, S. (2000). Using genetic algorithms to solve the motion planning problem. Journal of Universal Computer Science, 6(4), 422-432.

Jena, A., Sahu, P.K., Bharat, S.C., \& Biswal, B.B. (2016, July). Optimal trajectory planning of a 3R SCARA manipulator using geodesic. In 2016 IEEE 1st International Conference on Power Electronics, Intelligent Control and Energy Systems (ICPEICES), (pp. 1-6). IEEE.

Liu, H., Lai, X., \& Wu, W. (2013). Time-optimal and jerk-continuous trajectory planning for robot manipulators with kinematic constraints. Robotics and Computer-Integrated Manufacturing, 29(2), 309-317.

Olabi, A., Béarée, R., Gibaru, O., \& Damak, M. (2010). Feedrate planning for machining with industrial six-axis robots. Control Engineering Practice, 18(5), 471-482.

Rodnay, G., \& Rimon, E. (2001). Isometric visualization of configuration spaces of two-degrees-offreedom mechanisms. Mechanism and Machine Theory, 36(4), 523-545.

Sahu, P.K. (2016). Optimal trajectory planning of industrial robots using geodesic. IAES International Journal of Robotics and Automation, 5(3), 190-198.

Sahu, P.K., Balamurali, G., Mahanta, G.B., \& Biswal, B.B. (2019). A heuristic comparison of optimization algorithms for the trajectory planning of a 4-axis SCARA robot manipulator. In Computational Intelligence in Data Mining, (pp. 569-582). Springer, Singapore.

Selig, J.M., \& Ovseevitch, A.I. (1996). Manipulating robots along helical trajectories. Robotica, 14(3), 261267.

Tian, L., \& Collins, C. (2004). An effective robot trajectory planning method using a genetic algorithm. Mechatronics, 14(5), 455-470.

Yun, W.M., \& Xi, Y.G. (1996). Optimum motion planning in joint space for robots using genetic algorithms. Robotics and Autonomous Systems, 18(4), 373-393. 
International Journal of Mathematical, Engineering and Management Sciences

Vol. 4, No. 5, 1196-1207, 2019

https://dx.doi.org/10.33889/IJMEMS.2019.4.5-094

Zefran, M., Kumar, V., \& Croke, C.B. (1998). On the generation of smooth three-dimensional rigid body motions. IEEE Transactions on Robotics and Automation, 14(4), 576-589.

Zha, X.F. (2002). Optimal pose trajectory planning for robot manipulators. Mechanism and Machine Theory, 37(10), 1063-1086.

Zha, X.F., \& Chen, X.Q. (2004). Trajectory coordination planning and control for robot manipulators in automated material handling and processing. The International Journal of Advanced Manufacturing Technology, 23(11-12), 831-845.

Zhang, L., Zhou, C., \& Wang, D. (2007). Manipulator trajectory planning using geodesic method. In Robotic Welding, Intelligence and Automation, (pp. 517-528). Springer, Berlin, Heidelberg. 